\title{
Les transports sédimentaires sur les côtes sableuses, retour d'expérience sur la fiabilité des principales techniques de mesures
}

\author{
Franck Levoy ${ }^{1}$ et Olivier Monfort ${ }^{2}$
}

${ }^{I}$ Maître de conférences -HDR-, UMR 6143

"Morphodynamique Continentale et Côtière », Université de Caen, 02.31.56.57.44,levoy@geos.unicaen.fr

${ }^{2}$ Ingénieur d'études, UMR 6143

"Morphodynamique Continentale et Côtière », Université de Caen, 02.31.36.22.41, monfort@geos.unicaen.fr

\section{Résumé}

Différentes techniques complémentaires de mesures in situ permettent d'apprécier la direction et l'intensité du transport des sables. Chacune recèle des imprécisions sur la mesure elle-même, mais également sur les calculs qu'elle implique. Les suspensions peuvent être évaluées par des pièges sédimentaires ou des appareils optiques de mesure des concentrations. La valeur des résultats dépend surtout de la qualité de l'étalonnage des appareils. Mieux adaptés pour évaluer le charriage, les traceurs offrent une vision intégrée sur l'espace et le temps.

\begin{abstract}
$\underline{\text { Abstract }}$
Various complementary techniques allow obtaining in field appreciations of sand transport direction and rate. Each of them has inaccuracies due to the measurement itself but also to the necessary computations. The quality of suspension characteristics, obtained from sand traps and optical concentration sensors, depends on the instruments calibration. Best fitted for bed load processes, tracers result in a spatial and temporal integrated vision.
\end{abstract}

\section{Introduction}

Dans l'optique d'une meilleure compréhension de la mobilité des formes sédimentaires sur les littoraux, des mesures du transport des sédiments sont en général nécessaires. Si pour les sédiments fins, véhiculés en suspension, le prélèvement d'échantillons et l'enregistrement en parallèle des caractéristiques des courants permettent d'accéder aux flux sédimentaires, pour les sables, fréquemment transportés par charriage, le problème est plus délicat. Les mesures in situ à l'interface eau-sédiment sont en effet techniquement difficiles et sources de grandes imprécisions rendant parfois impossibles les interprétations en terme d'évolution des formes. La mesure des flux sédimentaires est néanmoins indispensable, notamment dans le domaine du génie côtier, pour disposer de 
données associant les caractéristiques hydrodynamiques et les transports associés afin d'améliorer la définition des formules de transports et de valider les modèles numériques d'évolution des fonds (Bodge et al., 1991).

La présente note vise à souligner les limitations des principales techniques de terrain utilisées pour quantifier les transports sableux et à orienter les interprétations des résultats grâce à leur prise en considération.

\section{Les techniques de quantification des déplacements sédimentaires : limites d'utilisation}

De nombreuses approches, directes ou indirectes, c'est-à-dire permettant de mesurer des taux de transports ou des concentrations, reposant sur l'expérimentation in situ, sont employées pour quantifier les déplacements sédimentaires sableux. Toutes présentent des avantages et des inconvénients qu'il convient de prendre en considération pour apprécier la validité des résultats obtenus et, par la même, leur fiabilité.

\subsection{Les mesures de concentration à l'aide de capteurs électro-optiques}

Des appareils qui utilisent l'influence des particules en suspension sur la propagation de la lumière (néphélomètres) sont couramment utilisés pour évaluer la turbidité en zone côtière. Les O.B.S. (Optical Backscatter Sensors) sont, par exemple, très populaires dans les études concernant la dynamique sédimentaire (Downing et al., 1981). Bien que de taille réduite, ces capteurs ne peuvent être positionnés très près des fonds (à moins de quelques centimètres) pour éviter que les affouillements et les remises en suspension qu'ils induisent ne faussent trop la mesure. Ils ne donnent donc pas accès au charriage et à la saltation. Comme tous les instruments dont la taille est voisine de la longueur caractéristique de variation des grandeurs physiques mesurées, ces capteurs sont de plus sensibles au bruit hydrodynamique. L'écoulement turbulent autour des appareils modifie, par effet d'inertie, la distribution spatiale des particules et tend donc à augmenter les gradients de concentration. Un filtrage fréquentiel permettrait de masquer ce défaut mais gommerait également les variations d'origine naturelle. La mesure est de plus intégrée sur un certain volume qui varie en fonction de la longueur de pénétration du rayon lumineux dans l'écoulement, elle-même liée à la concentration en matières solides en suspension. La discrimination entre ces artefacts et les variations naturelles est délicate, l'ordre de grandeur de ces erreurs étant intimement lié aux agents hydrodynamiques.

Après calibration, ces capteurs donnent accès à la turbidité exprimée par rapport à une certaine référence, le plus souvent N.T.U. (Nephelometric Turbidity Units) ou F.T.U. (Formazin Turbidity Units). Ces grandeurs sont relatives et sans grand intérêt pratique. Un étalonnage est nécessaire pour obtenir la concentration en Matières En Suspension (M.E.S.) qui est la grandeur physique recherchée. Il 
peut être réalisé a posteriori en comparant les concentrations présentes dans des prélèvements d'eau avec les valeurs mesurées au même moment par le néphélomètre (Battisto et al., 1999). Un prélèvement rigoureux, strictement dans le champ optique de la sonde, est toutefois délicat à réaliser.

Dans la pratique, cet étalonnage est souvent réalisé en cuve en enregistrant les mesures des capteurs de terrain pour différentes masses de sédiment, provenant $\mathrm{du}$ fond sous l'instrumentation, «artisanalement » remises en suspension (Thorne et al., 1991). Les installations ne permettent cependant pas de reproduire les conditions exactes de terrain : niveau de la lumière ambiante, concentration en matières dissoutes qui modifient la couleur de l'eau, présence de bulles. La composition du sédiment qui circule in situ devant les capteurs est par ailleurs le plus souvent différente de celle des particules présentes sur le fond sédimentaire et évolue, dans le temps et l'espace, avec les conditions hydrodynamiques. Les capteurs sont de plus sensibles à toutes les particules en suspension, vases, sables, mais aussi matières organiques, issues par exemple de «blooms » planctoniques, qui ne rentrent pas dans la problématique de l'étude des transports sédimentaires. La relation entre la réponse des O.B.S. et la concentration en M.E.S. dépend malheureusement largement de la taille, de la composition et de la forme des particules présentes devant les capteurs (Conner et De Visser, 1992). Il convient seulement de rappeler que le seuil de saturation d'un O.B.S. peut être atteint pour des concentrations de vases de l'ordre de quelques grammes par litre, alors que, pour des sables, des concentrations voisines de $100 \mathrm{~g} / \mathrm{l}$ seront inférieures à la valeur maximale mesurable. Les courbes d'étalonnage ainsi obtenues seront donc le plus souvent biaisées, voire approximatives.

La concentration obtenue $\mathrm{C}$ doit être combinée à la vitesse de l'écoulement $\mathrm{V}$ pour déterminer un flux sédimentaire $\mathrm{Q}$ selon l'expression: $\mathrm{Q}=\mathrm{C} \cdot \mathrm{V}$. Différents dispositifs, permettant l'acquisition en parallèle de ces deux caractéristiques, sont d'ailleurs disponibles sur le marché. L'intégration sur la hauteur de la tranche d'eau, basée sur des hypothèses concernant les profils verticaux de vitesse et de concentration, permet par la suite d'aboutir à un débit solide instantané par unité de longueur suivant une direction normale à l'écoulement. La mesure simultanée des courants et des concentrations à plusieurs niveaux sur la verticale facilite cette intégration sur la colonne d'eau (Miller et al., 1999).

\subsection{Les pièges à sédiments}

Ils permettent une mesure directe des flux sédimentaires sur l'avant-côte (Salomon, 1976; Chapalain et Thais, 2001) et les plages (Kraus, 1987; Rosati et al., 1991). Il en existe de nombreuses variantes reposant toujours sur le même principe, à savoir : le déploiement sur une structure, installée sur les fonds marins ou mise en œuvre à partir d'un bateau, de réceptacles destinés à piéger les sédiments en mouvement. Leur déploiement peut s'avérer délicat dans les zones à haute énergie, en présence de courants intenses ou dans le déferlement par 
exemple. Comme pour tout instrument de mesure, la condition première à l'utilisation des pièges est que leur présence ne perturbe pas trop le phénomène à quantifier, c'est à dire les transports solides dans le cas présent. D'un point de vue pratique, cette condition est évidemment impossible à satisfaire. Le simple fait d'introduire un objet dans l'écoulement modifie en effet, au moins localement, la direction et la vitesse des courants et donc le transport solide. L'affouillement localisé sous la structure, d'autant plus marqué que les réceptacles sont proches $\mathrm{du}$ fond, témoigne notamment de cette influence.

La plupart du temps, les pièges sont testés dans des canaux pour apprécier la modification du champ de courants à leur voisinage et dans l'entrée du réceptacle (Rosati et Kraus, 1989). Il en ressort des courbes correctrices, mettant en relation la vitesse du courant avec et sans l'instrument, qui laissent présager de l'influence du piège sur les transports sédimentaires. Rares sont en revanche les études qui considèrent le caractère évolutif de cette influence. La perturbation de l'écoulement par le piège s'accentue en effet au fur et à mesure du remplissage des réceptacles. En d'autres termes, la modification du champ de courant induite par le piège est éminemment liée à la durée d'immersion du dispositif, aux caractéristiques de l'écoulement et du débit solide. Aucune loi correctrice standardisée ne permet en réalité d'évaluer le flux sédimentaire, qui aurait été observé en l'absence du piège, à partir du matériel stocké.

Les matériaux accumulés dans les réceptacles, une fois séchés et pesés, permettent le calcul du flux sédimentaire moyen pour la hauteur correspondante par rapport au fond. L'utilisation de plusieurs réceptacles, disposés l'un au-dessus de l'autre, donne une idée de la répartition verticale des flux et permet une intégration sur la tranche d'eau pour aboutir à un débit solide pour une longueur unitaire dans la direction d'ouverture du réceptacle. Des hypothèses simplificatrices, concernant le profil de transport entre le fond et la surface libre, doivent pour cela être faites. Le nombre et la disposition des réceptacles sur la verticale résultent d'un compromis pour réduire les erreurs de mesure et les incertitudes liées aux calculs. L'augmentation du nombre des réceptacles améliore la définition du profil vertical des transports mais, en contrepartie, accentue les perturbations induites sur l'écoulement. La hauteur des réceptacles par rapport au fond est une donnée importante qui peut être difficile à évaluer (zones à haute énergie ou déploiement à partir de navires) et évoluer au cours du temps avec la modification du régime hydrodynamique. En disposant plusieurs réceptacles l'un à côté de l'autre de manière à couvrir un secteur de quelques dizaines de degrés à $360^{\circ}$, il est possible d'évaluer un débit sédimentaire résiduel. Les interpolations nécessaires sont, de la même manière que l'intégration des flux sur la verticale, sources d'imprécisions.

\subsection{Les traceurs fluorescents et radioactifs}

Le traceur, qu'il soit naturel ou artificiel (sables peints pour les traceurs fluorescents et billes de verre irradiées pour les traceurs radioactifs), préparé et 
déposé sur estran ou en mer est suivi pendant une durée qui peut varier de quelques heures à plusieurs mois. Réputées plus adaptées à la quantification des charriages que des suspensions, ces techniques, basées sur le déplacement du centre de gravité du nuage de traceur, sont limitées à l'étude des transports par advection. Les phénomènes de diffusion (déplacement du traceur dû à des mouvements aléatoires moléculaires ou turbulents) et de dispersion qui n'induisent pas un déplacement du centre de gravité du nuage ne sont pas correctement mesurés par ces approches. Le transport obtenu est intégré dans le temps et l'espace (Ingle, 1966 ; Courtois et Monaco, 1969 ; Caillot et al., 1978 ; White et Inman, 1989).

Pour être utilisable comme marqueur, le matériel adopté doit avoir un comportement dynamique aussi proche que possible de celui du stock sédimentaire dont les déplacements doivent être étudiés. Ils doivent être similaires du point de vue de la densité, de la rugosité de surface, du spectre granulométrique, du triage et des caractéristiques de forme (le comportement des fractions coquillières aplanies est différent de celui de particules siliceuses le plus souvent arrondies).

Outre les difficultés liées à la lourdeur de ces expérimentations (fabrication, détection de nuit des traceurs fluorescents, obtention des autorisations et contraintes de maniement des traceurs radioactifs, ...), ces techniques reposent aussi sur des hypothèses, nécessaires au traitement des données, qui mériteraient d'être affinées. L'une d'entre-elles concerne la détermination des limites de la tâche fluorescente ou radioactive dont l'imprécision peut fausser l'estimation des flux (domaine d'intégration inexact). L'évaluation de l'épaisseur de remaniement du matériel, qui permet de calculer le volume de sédiment déplacé, est une autre source potentielle d'incertitude. Pour les traceurs fluorescents, cette épaisseur est en général assimilée à la profondeur maximale de pénétration dans la colonne sédimentaire des particules marquées. Selon le domaine considéré (plage ou bord de chenaux en domaine estuarien ouvert par exemple) et la morphologie de détail du lit sédimentaire (présence ou absence de rides), l'épaisseur de remaniement peut varier dans le temps et l'espace. Les traceurs radioactifs fournissent indirectement une vision de la variabilité spatiale de cette caractéristique au moment de la détection. Son évolution entre deux détections est par contre inaccessible. D'autres approximations sont habituellement faites, particulièrement dans le cas des traceurs fluorescents (Levoy et al., 1997): épaisseur de remaniement uniforme, identité entre la concentration du traceur en surface et dans le volume (dans le cas des comptages de surface), évaluation de la concentration à partir du comptage d'un nombre de grains, hypothèses simplificatrices retenues pour l'intégration. Elles peuvent conduire à des résultats parfois surprenants : la masse de traceur au moment de la détection, obtenue par intégration sur la surface de la tache, est parfois supérieure à la quantité immergée. 


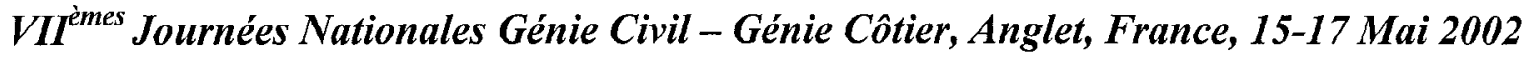

\section{Discussion}

Les techniques intrusives, utilisant des pièges à sédiments ou des capteurs electro-optiques, modifient nécessairement les écoulements et donc les flux sédimentaires. L'étalonnage de ces dispositifs, souvent délicat et coûteux, conditionne directement la valeur des résultats obtenus par ces méthodes. Ces approches, de type eulérien, ne permettent pas d'appréhender les transports par charriage ou saltation pour lesquels les mesures doivent être réalisées dans la couche mobile à l'interface entre le fond et l'écoulement. Les pièges à sédiments donnent une information qui peut être quasi ponctuelle mais qui est intégrée sur la durée de l'expérience. Les néphélomètres donnent pour leur part des résultats ponctuels et instantanés.

Les pièges doivent de préférence être utilisés dans des conditions de faible énergie et pour des expériences de relativement courte durée (quelques dizaines de secondes à quelques dizaines de minutes). La durée d'immersion doit cependant être ajustée à l'intensité des transports sédimentaires. La possibilité de contrôler le remplissage du piège et l'optimisation de leur géométrie pour réduire les perturbations qu'ils induisent permettront d'améliorer la qualité des résultats. Malgré leur relative commodité, ils ne permettent pas d'obtenir des transports sableux en zone littorale très fiables, d'un point de vue absolu. L'efficacité du piège (rapport entre le débit déduit du matériel stocké dans les réceptacles et le transport qui aurait pu être observé en l'absence de la structure) est largement fonction des perturbations induites par l'instrument, elles-mêmes liées aux conditions d'écoulement et à l'intensité des transports. Au mieux, cette technique permet d'apprécier de manière relative la distribution des flux sédimentaires sur la verticale ou par secteur.

L'étalonnage des instruments de mesure à haute fréquence des teneurs en M.E.S. est un problème toujours d'actualité. La taille des particules et la teneur en matières organiques en suspension peuvent influencer les valeurs brutes enregistrées. Les particules fines sont le plus souvent présentes sous forme de nuages uniformes et peu sensibles à l'influence de vagues individuelles tandis que la mise en suspension des sables se fait par intermittence avec des échelles de temps caractéristiques inférieures à celles de mouvements orbitaux. La soustraction du signal d'un bruit de fond permet alors de filtrer l'influence des matières fines (Battisto et al., 1999). L'immersion d'un granulomètre laser, comme sur le site de Duck en Caroline du Nord (USA), et l'utilisation de dispositifs automatiques de pompage permettent également d'appréhender la variabilité temporelle du spectre granulométrique (Battisto et al., 1999). Ces solutions ne permettent cependant pas de filtrer l'influence des matières organiques et nécessitent de nombreux prélèvements et analyses, au moins à chaque modification majeure des conditions hydrodynamiques. Elles accentuent le caractère intrusif de l'instrumentation et restent lourdes, coûteuses et donc peu courantes. Les erreurs liées au niveau de fond de turbidité et à la position des capteurs induisent des incertitudes sur les transports solides qui s'élèvent, selon 
Miller et al. (1999), à $10 \%$ et $15 \%$ respectivement. Les flux sédimentaires doivent avant tout être considérés de manière relative, les concentrations en M.E.S. obtenues étant directement liées à la qualité de l'étalonnage des capteurs et aux conditions de site difficiles à percevoir au travers des simples résultats.

Les traceurs donnent pour leur part accès aux transports qui s'effectuent à proximité immédiate du fond. Cette approche lagrangienne est par ailleurs préférable du point de vue des perturbations induites sur le milieu. Elle permet d'estimer des débits solides intégrés sur le temps, entre deux détections, et l'espace, sur la surface de la tache, et sont donc le reflet de l'action intégrée de conditions hydrodynamiques, elles aussi variables temporellement et spatialement. Plus la durée entre 2 détections sera longue et plus les agents à l'origine des mouvements pourront avoir varié, rendant difficile la discrimination de l'influence respective de chacun. Sur un estran découvrant, par exemple, les déplacements peuvent traduire l'action des houles déferlantes, des courants de marée et parfois même des transports éoliens à marée basse, ces phénomènes pouvant agir simultanément ou à des instants différents du cycle tidal. Malgré les hypothèses simplificatrices qu'elle suppose, cette technique donne le plus souvent une bonne idée du mouvement moyen des sédiments sur le site considéré et la durée de l'expérimentation. L'estimation de la direction du transport sera, d'un point de vue qualitatif, bien meilleure que celle de l'intensité.

\section{Conclusion}

Il existe donc une réelle difficulté à réaliser in situ des mesures de transports sédimentaires fiables à mettre en parallèle des facteurs hydrodynamiques responsables de ces mouvements. Celles-ci sont souvent imprécises à l'image d'ailleurs des résultats issus des calculs reposant sur des formulations semiempiriques. L'aspect intrusif de certaines méthodes, la complexité des mélanges «sables-vases-matières organiques » en mouvement, les hypothèses retenues pour le traitement des données expliquent ces imprécisions. D'un point de vue technique, la mesure fine au niveau de la couche-limite (couche fluidisée en mouvement à l'interface) semble difficilement envisageable in situ. Ces lacunes empêchent actuellement de progresser sur la définition de formules de transports véritablement adaptées à l'environnement côtier sableux et notamment à la prévision de l'évolution des plages et du trait de côte. De fait, les modèles numériques morphodynamiques, utilisant les formules actuellement disponibles, ne peuvent simuler, avec une réelle fiabilité, l'évolution des fonds sédimentaires, ceci d'autant plus sur le long terme. Les ordres de grandeurs, concernant l'intensité et la direction des mouvements sédimentaires, obtenus par les méthodes classiques de mesure, s'avèrent néanmoins satisfaisants et du plus grand intérêt dans le domaine de l'aménagement du littoral et du génie côtier, notamment pour évaluer l'aspect qualitatif des impacts de la construction d'ouvrages. L'interprétation des résultats devra néanmoins se faire avec prudence et tenir compte des incertitudes liées aux mesures elles-mêmes et aux calculs nécessaires. 


\section{Références}

Bodge, R.K., Kraus, C.K. (1991) - Critical examination of longshore transport rate magnitude. Coastal Sediments'91, 139-155.

Battisto, G. M., Friedrichs, C.T., Miller, H.C., Resio, D.T. (1999) - Response of OBS to mixed grain-size suspensions during Sandyduck'97. Coastal sediments'99. vol. 1, 297-312.

Caillot, A., Quesney, M., Wilson, G. (1978) - Contribution à l'étude de mouvements sédimentaires le long du littoral belge à l'aide de traceurs radioactifs. Mesure du débit de charriage. Rapport C.E.A. 13/ C 256, 48 p.

Chapalain, G., Thais, L. (2001) - A system for measuring horizontal sand transport by currents. Journal of Coastal Research, 17 (1), 162-172.

Conner, C.S., De Visser, A.M. (1992) - A laboratory investigation of particule size effects on Optical Backscatterance sensor. Marine Geology, 108, 151-159.

Courtois, G., Monaco, A. (1969) - Radioactive methods for the quantification of coastal drift rate. Marine Geology, 7, 183-206.

Downing, J.P. Sternberg, R.W., Lister, C.R.B. (1981) - New instrumentation for investigation of sediment suspension processes in the shallow marine environment. Marine Geology, 42, 19-34.

Ingle, J.C. (1966) - The mouvement of beach sand. An analysis using fluorescent grain. Developments in sedimentology, 5, $211 \mathrm{p}$.

Kraus, N.C. (1987) - Application of portable traps for obtaining point measurements of sediment transport rates in the surf zone. Journal of Coastal Research, 2, 139-152.

Levoy, F., Monfort, O., Larsonneur, C. (1997) - Quantification des débits solides sur les plages macrotidales à l'aide de traceurs fluorescents, application à la côte ouest du Cotentin. Océanologica Acta, vol. 20, $\mathrm{n}^{\circ}$ 6, 811-822.

Miller, H.C., Smith, S.J., Hamilton, D.G., Resio, D.T. (1999) - Cross-shore transport processes during onshore bar migration. Coastal sediments'99, vol. 2, 1065-1080.

Rosati, J.D., Kraus, C.K. (1989) - Development of a portable sand trap for use in the nearshore. Department of the army, U.S. Corps of Engineers. Technical report CERC-89-11, $181 \mathrm{p}$.

Rosati J.D., Gingerich K.J. Kraus N.C., Smith J.McK., Beach R.A. (1991) Longshore sand transport rate distribution measured in Lake Michigan. Coastal Sediment '91, vol. I, 156-169.

Salomon, J.C. (1976) - Modèle mathématique de la propagation de la marée en estuaire et des transports sableux associés. Application aux estuaires de la Loire et de la Seine. Thèse de doctorat, Université de Brest, $257 \mathrm{p}$.

Thorne, P.D., Vincent, C.E., Hardcastle, P.J., Rehman, S., Pearson, N. (1991) Measuring suspended sediment concentrations using acoustic backscatter devices. Marine Geology, 98, 7-16.

White, T.E. et Inman, D.L. (1989) - Measuring longshore transport with tracers. Seymour Editor, 287-312. 